\title{
Incentives and the Structure of Teams
}

\author{
April Franco*, Matt Mitchell ${ }^{\dagger}$ and Galina Vereshchagina ${ }^{\ddagger}$
}

January 2008

\section{PRELIMINARY AND INCOMPLETE}

\begin{abstract}
This paper endogenizes the matching structure of teams in a simple environment with moral hazard. We show that team incentive problems may, on their own, generate monotone matching predictions in the absence of complementarities or anti-complementarities in production technology. We also derive sufficient conditions on the primitives of the model leading to the optimality of positive and negative matching of team members.
\end{abstract}

\footnotetext{
${ }^{*}$ Rotman School of Management, University of Toronto

${ }^{\dagger}$ Rotman School of Management, University of Toronto

$¥$ Arizona State University
} 


\section{Introduction}

Team production and team performance-based compensation schemes have been adopted by many firms over the last 30 years (e.g. Hamilton et al. 2003). Given that employees usually differ in their skills and productivity, it is important to understand what kind of teams would maximize firms' profit. Namely, should the high skilled workers be teamed with high skilled or low skilled partners? This question is the main focus of our paper.

It is well known from the matching literature that the matching patterns crucially depend on the properties of the payoff function: in the frictionless world with transferable utility supermodularity leads to positive matching, while submodularity induces negative matching (e.g., Becker (1973)). Many models assume that the technology has some complementarity structure which the payoff function inherits (e.g. Kremer (1993)). By contrast, we study an environment where the technology is neither supermodular nor submodular, and show that predictions about matching structure can arise purely because of the team incentive problem.

Our results are derived in a standard model of moral hazard, in which a profit maximizing planner (firm owner) decides how to organize agents (workers) into teams. The team output depends on the efforts exerted by the team members. Workers differ in their productivity (or the cost of exerting effort). The planner observes the types of the workers, but not the actual exerted effort. The production technology is chosen in such a way that, under complete information, the planner is indifferent between the possible matching structures. In order to make the results as plain as possible, we focus on a very stark model, in which the planner has to form two teams of two out of four workers, given that two of the workers have high productivity and the other two are less productive.

Then we develop three sets of results concerning the optimal matching structure when efforts are not observable. First, we show that when types are ordered by the level of input they provide, the ordering of rewards among those types determines the optimal matching structure. In particular, if higher input types are rewarded more in the event of a success, then negative matching is optimal. If higher input types are rewarded less, then the teams should be matched positively. 
The intuition is that the team structure generates payments that can be broken into two categories. On the one hand, the agent may be paid for a success for which his effort was responsible. On the other hand, he may be also paid as a result of the effort of his teammate. We call this second kind of payment an "accidental" payment. To minimize the costs associated with accidental payments, the firm owner should team the high input worker with the one whose rewards are low, so that frequently paid accidental compensations are small in magnitude. Correspondingly, if the high input worker is promised a relatively low reward, positive matching is optimal; if, on the contrary, high input workers are paid high rewards, it is better to form mixed teams.

Then we go on to develop results about the impact of the shape of the underlying technology on the optimal matching structure. As standard in the literature, incentive compatibility stipulates a particular relationship between the effort requested by the firm owner and the reward promised to the worker. This relationship is determined only by the properties of exogenous production technology. We show that, when input levels are interior, positive matching is associated with cases where the reward required to implement a particular effort level is a convex function of effort, while negative matching becomes optimal if the reward function is sufficiently concave.

This result is related to the first one, about the relationship between rewards and efforts. Consider two agents, one with lower cost. It is natural (and, indeed, optimal), for the agent with lower cost to be made to give more effort. But since that agent has lower cost, a particular level of effort can be achieved with a smaller reward. The question, then, is how much greater a level of effort should be chosen for the low cost agent, and how much more cost that higher effort will necessitate. When the required reward is a very convex function of effort, it is prohibitively expensive to make effort substantially higher for the low cost agent, and therefore the main difference between the agents is not effort levels, but the required cost to provide a given effort level. As a result, the higher input agent (the low cost type) is paid less in the event of a success, and positive matching is optimal. By contrast, when the reward required to implement a given level of effort is a concave function, the low cost agent can be asked to give a great deal higher effort, overcoming the lower cost and implying that the low cost agent gets a greater reward in the event of a success. The first set of results implies that 
this case is exactly the one where negative matching is optimal.

Our final set of results completely characterizes the matching structure for an exponential functional form for the production technology. The case is one where the previous results imply positive matching for interior cases. However, we show how matching can go from positive to negative as parameters move into the region where corners become relevant. When the low skill type becomes sufficiently inefficient, it is optimal to eventually not to elicit effort from the low skill types, and instead spread out the high skill types across projects to mitigate team incentive problems.

We also discuss three extensions. First, we consider increasing the team size. We argue that increases in team size will tend to lead to corners that in turn generate negative matching, sometimes in cases where positive matching was optimal for smaller teams. The intuition is related to the idea of accidental payments: having more team members generates more accidental payments, and therefore creates an incentive to lower some members' reward (to zero) in order to keep accidental payments small.

Finally, we consider alternative ways of modeling the incentive problem. First, our model uses limited liability and risk neutral agents to generate stark results. We show numerically that similar results can be obtained in a model with risk averse agents and no limited liability. We argue that such an environment also introduces a variety of other effects that make it analytically intractable. Second, our results are derived in the environment in which the firm owner organizes the workers in teams with the objective of maximizing own profit. We also consider an alternative model in which the workers form partnerships and agree to split the team's output between themselves. This formulation is also not tractable analytically, even for particular functional forms. Thus we perform a series of numerical exercises, and find that, as in our benchmark model, the properties of the underlying technology may similarly lead to either positive or negative matching patterns in partnership formation.

Our work is closely related to two strands of literature. First, moral hazard has been extensively discussed in the literature as a natural feature of team production. A large number of studies have analyzed the implications of the free-riding incentives as well as the properties of the optimal compensation to the team members (e.g., Holmstrom (1982), McAfee and McMillan (1991)). To our 
knowledge, however, our paper is the first attempt to understand whether moral hazard in teams may have certain implications for the optimal team structure in the presence of worker heterogeneity.

Second, there has been a lot of work done, dating back to Becker (1973) focusing on matching patterns among heterogeneous agents. Recently, a number of studies analyzed how matching predictions might be affected by various economic frictions. For instance, one line of research pioneered by Shimer and Smith (2000) focuses on the role of search frictions and argues that in search models positive assortative matching may fail even if the joint production function is supermodular. Another friction that is likely to weaken the effects of technological complementarities is described by Kaya (2008), who illustrates that if the types of the matching partners are not observable, some of the low-type agents cannot be deterred from mimicking the high-type agents and, therefore, positive assortative matching cannot be sustained in the equilibrium. Our paper studies a different friction (moral hazard) and obtains quite different results: in contrast to the findings discussed above, we show that, depending on the parameters of the model, moral hazard can either reduce or decrease the degree of technological complementarity embodied in production technology.

The effects of moral hazard on matching predictions have been investigated by Thiele, Wambah (1998) and Newman (2007) in an environment different from ours. They study a problem of assigning heterogeneous in wealth, risk averse entrepreneurs to the projects with different amount of risk. In the absence of frictions, wealthier (and hence less risk averse) entrepreneurs would undertake riskier projects. However, if the entrepreneur's effort is unobservable, the opposite matching pattern may arise if the utility is linear in effort. This is because richer agents have lower marginal utility of income, and should be offered higher compensation to induce a particular effort level. While our paper also emphasizes the role of moral hazard, our question, as well as modeling environment, is very different from theirs, and the mechanism outlined in these papers does not play any role in generating our results.

Finally, Prat (2002) poses a question related to ours (how to organize workers in teams?), but he studies the effects of a different friction. The paper introduces learning into a team production model and shows that, in spite of this modification, the matching patterns are still determined by the super- or sub- modularities 
in the production technology. In contrast, in our model all the matching predictions arise solely due to moral hazard friction and are not driven by the properties of the underlying technology.

\section{Model}

Consider a technology owned by a risk neutral principal that requires two agents to be operable. Output from the technology is stochastic and depends on the input from each agent $\theta_{i}$. Denote that input by $x_{i}$. The output is (by normalization) one with probability $g\left(x_{1}\right)+g\left(x_{2}\right)$, where $g(x)$ is increasing and satisfies $0 \leq g(x)<1 / 2$. Otherwise output is zero. We will term output of one a "success."

Our choice of functional form for the success probability is driven by two considerations. First, we want the choice to be such that, in the absence of information frictions, matching is irrelevant. That is satisfied because of the additive separability in the inputs $x_{1}$ and $x_{2}$, as we will show formally below. Second, we want the function to be such that there is always some chance of failure, and therefore the incentive problem will be unavoidable when inputs are unobserved. This motivates the admissible range for $g(x)$.

Each agent is risk neutral and has cost of effort $c(x, \theta)$, where $\theta$ is the agents type. The agent has limited liability, in the sense that state by state, the wages paid cannot be lower than zero. We discuss relaxing the limited liability constraint below. Without loss, we can restrict attention to contracts that pay zero for failures and $w_{\theta} \geq 0$ for successes.

In order to address the question of whether to choose homogeneous or heterogeneous teams, we will suppose that the principal operates two teams and is faced with four agents, two each of types $\theta=l$ and $\theta=h$, respectively. The principal then has to decide whether to match like types or different types.

\section{Benchmark: Complete Information}

A planner who could observe the inputs $x$ could simply choose $x_{\theta}^{*}$ for each agent to maximize

$$
g\left(x_{\theta}^{*}\right)-c\left(x_{\theta}^{*}, \theta\right)
$$


and pay

$$
w_{\theta}=c\left(x_{\theta}^{*}, \theta\right) /\left(g\left(x_{\theta}^{*}\right)+g\left(x_{\theta^{-}}^{*}\right)\right.
$$

where $\theta^{-}$is the type of the agent's teammate. Four agents, then ,would generate expected output of $2 g\left(x_{l}^{*}\right)+2 g\left(x_{h}^{*}\right)$ and be paid expected wages of $2 c\left(x_{l}^{*}, l\right)+$ $2 c\left(x_{h}^{*}, h\right)$ regardless of the matching structure.

\section{Incomplete Information}

In the rest of the paper we focus on the case where only success or failure is observable, but inputs are not. The principal chooses a team structure, either matching like types (positive matching) or different types (negative matching). He chooses efforts $x_{\theta}$ and wages $w_{\theta}$ in a way that satisfies the incentive constraint

$$
x_{\theta} \in \arg \max _{x} g(x) w_{\theta}-c(x, \theta)
$$

An important feature of our additive structure for the underlying technology is that this incentive constraint is valid regardless of the team structure. The agent also collects $g\left(x_{\theta^{-}}\right) w_{\theta}$, but that is not relevant to the choice of $x$, and hence is left out of the incentive constraint. In other words, the agent's partner plays no role in the provision of incentives. Below we discuss alternative formulations where this is not the case, and argue that the intuition we develop for this stark model will naturally carry over.

The principal collects all output, net of wage payments. Formally, the principal can choose either to match positively, in which case he solves

$$
\begin{aligned}
\max _{x_{h}, x_{l}, w_{h}, w_{l}} 2 g\left(x_{h}\right)\left(1-2 w_{h}\right)+2 g\left(x_{2}\right)\left(1-2 w_{l}\right) \\
\text { s.t } \\
x_{\theta} \in \arg \max _{x} g(x) w_{\theta}-c(x, \theta), \theta \in h, l
\end{aligned}
$$

or negatively, in which case he solves 


$$
\begin{aligned}
& \max _{x_{h}, x_{l}, w_{h}, w_{l}} 2\left(g\left(x_{h}\right)+g\left(x_{l}\right)\right)\left(1-w_{h}-w_{l}\right) \\
& \text { s.t } \\
& x_{\theta} \in \arg \max _{x} g(x) w_{\theta}-c(x, \theta), \theta \in h, l
\end{aligned}
$$

\subsection{Payment vs. Inputs}

We first establish the following basic result relating matching patterns with the correlation between effort and reward specified by the optimal contract.

Claim 1 Negative (positive) matching can be the optimal choice of the planner only if for such matching structure high-input types receive high (low) compensation in the event of success.

Proof. Suppose to contradiction that the planner chooses to sort workers positively and offers higher compensation to the types exerting more effort. We will show that the planner's profit would increase if, instead, the workers were rematched negatively.

For brevity, denote $g\left(x_{l}\right)$ and $g\left(x_{h}\right)$ by $g_{l}$ and $g_{h}$. The total surplus of the firm owner matching his teams positively is equal to

$$
\Pi^{+}=2 g_{l}\left(1-2 w_{l}\right)+2 g_{h}\left(1-2 w_{h}\right)
$$

As was noted above, the same contract will still be incentive compatible if the teams are rematched negatively (since IC constraint (1) is affected only by the worker's own type). By rematching the workers negatively, and offering the same contract, the firm owner would obtain profit

$$
\Pi^{-}=2\left(g_{l}+g_{h}\right)\left(1-w_{l}-w_{h}\right)
$$

It is easy to verify that

$$
\Pi^{-}-\Pi^{+}=2\left(g_{l}-g_{h}\right)\left(w_{l}-w_{h}\right),
$$


which is positive if high-input workers receive high rewards. Hence, the planner would increase his expected profit by rematching the workers.

The opposite result is based on a symmetric argument.

This result relates an endogenous (but possibly empirically observable variable), relative pay, to the matching structure. The intuition is straightforward. Agents make "earned" income $g\left(x_{\theta}\right) w_{\theta}$ and "accidental" income $g\left(x_{\theta^{-}}\right) w_{\theta}$. To keep accidental income at a minimum, agents with high inputs (i.e. high $x$, and therefore high $g(x))$ should be matched with agents with low wages. This insures that the relatively likely accidental payments are kept as small as possible.

The result shows that matching may be irrelevant only if, for any matching structure, either both types provide the same input or both types receive the same wages. ${ }^{1}$ In general, differences in cost will generate different optimal inputs, and different inputs will require different payments, given the differences in costs. In other words, typically there will be a non-trivial matching problem.

Notice that the statement of Claim 1 is conditional on the properties of the variables that are endogenously determined within our model, and it cannot be immediately stated how the efforts and rewards should be related to each other in the optimal incentive compatible contract. The reason is that the workers with the higher cost of effort should, on one hand, obtain a higher compensation for every unit of effort they provide, but, on the other hand, would also be requested to exert lower effort level. These two effects act in the opposite directions and make the relationship between efforts and rewards ambiguous. In the following section we develop a simple example illustrating that, depending on the properties of $g(x)$, either of the two effects may dominate, implying that moral hazard may potentially generate either positive or negative matching.

\subsection{The shape of $g$ and matching: an example}

To illustrate that the shape of $g(x)$ may be related to the optimal matching structure, consider the following example. Suppose that $c(x, \theta)=\theta x$ with $\theta \in$ $\left\{\theta_{l}, \theta_{h}\right\}$ and $\theta_{l}<\theta_{h}$. Let $g(x)$ be piecewise linear, with slope $a$ for low $x$, slope

\footnotetext{
${ }^{1}$ Notice that the matching structure becomes irrelevant if, for instance, effort is bounded from above and all the workers are asked to exert the maximum possible effort. The same would be true if the effort choice is discrete - work or do not work - and the optimal contract induces everyone to work.
} 


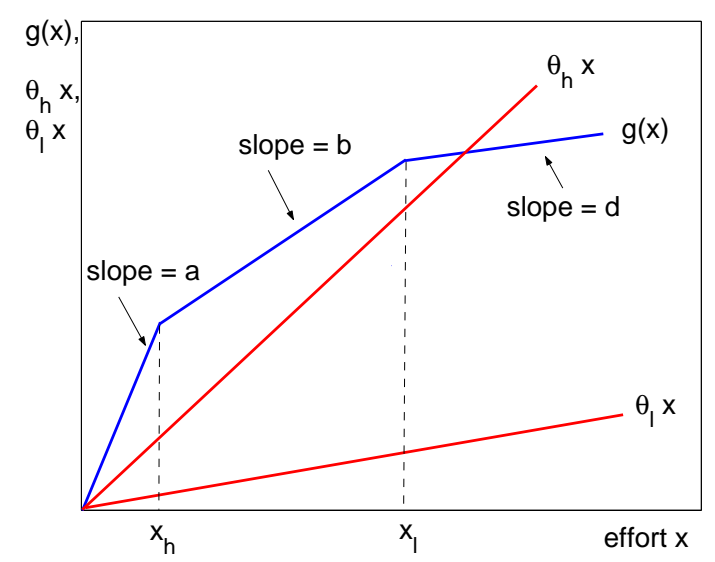

Figure 1: An example with piecewise linear $g(x)$.
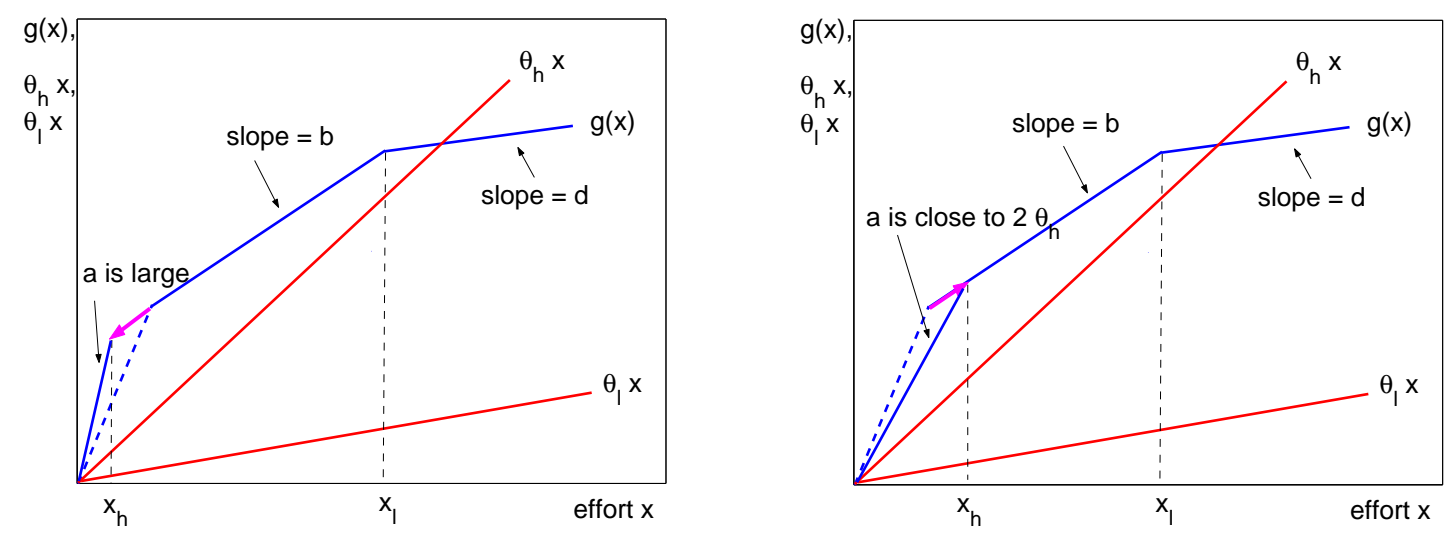

Figure 2: Adjusting $a$ and $x_{h}$ to achieve negative matching (on the left) and positive matching (on the right)

$b<a / 2$ for intermediate values of $x$ and slope $d<b / 2$ for large $x$. Suppose also that $a / 2>\theta_{h}>b$ and $b / 2>\theta_{l}>d$. An example of such $g(x), \theta_{l} x$ and $\theta_{h} x$ is illustrated on Figure 1. Notice that it is always optimal to set $x_{h}$ at the lower kink point and $x_{l}$ at the higher as it is illustrated on Figure 1. The corresponding payments should be $w_{h}=\theta_{h} / a$ and $w_{l}=\theta_{l} / b .^{2}$

To see why this shape of $g(x)$ may endogenously lead to any matching structure, consider adjusting $x_{h}$, while holding fixed $x_{l}, g\left(x_{l}\right)$, and $b$. The left plot of

\footnotetext{
${ }^{2}$ Note that $a / 2>\theta_{h}>b$ and $b / 2>\theta_{l}>d$ imply that the planner's expected payoff is positive for either matching structure. This guarantees that the firm owner would not shut any of the teams down. It is still possible, however, that shutting one of the workers down would generate higher payoffs. Additional conditions may be imposed on the parameters to eliminate such possibility.
} 
Figure 2 gives an example of function $g(x)$, for which negative matching is optimal: when $x_{h}$ is sufficiently low and, correspondingly, $a$ is sufficiently high, the agent who contributes less receives smaller payment, since $w_{h}=\theta_{h} / a$ becomes smaller than $w_{l}=\theta_{l} / b$. If, on the other hand, $x_{h}$ is sufficiently high so that $a$ is arbitrary close to $2 \theta_{h}$ (as illustrated on the right plot), the payment to the high-cost agent becomes bigger than the payment to the lower-cost agent, and positive matching becomes optimal.

\subsection{Interior solutions}

Suppose that $g(x)$ is concave and three times differentiable, and, as in the previous section, the cost function for type $\theta$ is $c(x, \theta)=\theta x .^{3}$ In this case the incentive constraint for any interior effort level simplifies to

$$
g^{\prime}\left(x_{\theta}\right) w_{\theta}=\theta
$$

We can rewrite this as

$$
w_{\theta}=\theta / g^{\prime}(x),
$$

or, letting $r(x)=1 / g^{\prime}(x)$,

$$
w_{\theta}=\theta r(x)
$$

We interpret $r(x)$ as the shape of the reward as a function of effort.

Note that for $h>l$, it is easy to show that any optimal contract must have $x_{h}<x_{l}$, or else the agents should reverse inputs. To see how the shape of $r(x)$ is related to the matching structure, suppose there is negative matching. We can use the first order condition to write the principal's payoff just as a function of the inputs:

$$
\left(g\left(x_{l}\right)+g\left(x_{h}\right)\right)\left(1-\theta_{l} r\left(x_{l}\right)-\theta_{h} r\left(x_{h}\right)\right)
$$

The first order conditions are

$$
\begin{aligned}
g^{\prime}\left(x_{l}\right)\left(1-\theta_{l} r\left(x_{l}\right)-\theta_{h} r\left(x_{h}\right)\right) & =\theta_{l} r^{\prime}\left(x_{l}\right)\left(g\left(x_{l}\right)+g\left(x_{h}\right)\right) \\
g^{\prime}\left(x_{h}\right)\left(1-\theta_{l} r\left(x_{l}\right)-\theta_{h} r\left(x_{h}\right)\right) & =\theta_{h} r^{\prime}\left(x_{h}\right)\left(g\left(x_{l}\right)+g\left(x_{h}\right)\right)
\end{aligned}
$$

\footnotetext{
${ }^{3}$ If $c(x, \theta)$ is linear in $\theta$, i.e. $c(x, \theta)=\theta \widetilde{c}(x)$, it is without loss of generality to assume that $c(x, \theta)=\theta x$, because it is always possible to redefine the variables in such a way that either $g(x)$ or $\widetilde{c}(x)$ is linear in $x$.
} 
So we have that

$$
\frac{g^{\prime}\left(x_{l}\right)}{g^{\prime}\left(x_{h}\right)}=\frac{\theta_{l} r^{\prime}\left(x_{l}\right)}{\theta_{h} r^{\prime}\left(x_{h}\right)}
$$

or

$$
\frac{\theta_{h} r\left(x_{h}\right)}{\theta_{l} r\left(x_{l}\right)}=\frac{r^{\prime}\left(x_{l}\right)}{r^{\prime}\left(x_{h}\right)}
$$

Since this is a negative match, the left hand side must be less than one, as it is the ratio of the pay of the low input type to the high input type. However, the right hand side can't be less than one if $r$ is convex and $x_{l}>x_{h}$. So convex $r(x)$ implies that we could not have it be optimal to match negatively. Put in terms of $g(x)$, we have the following result:

Claim 2 Suppose that $-\frac{g^{\prime \prime}(x)}{\left(g^{\prime}(x)\right)^{2}}$ is (weakly) increasing and that in the mixed teams the solution is interior. Then it is optimal to sort the teams positively.

A variety of commonly used examples meet this sufficient condition, including $g(x)=.5\left(1-e^{-x}\right), g(x)=A-B / x, g(x)=x-A x^{2}$, and $g(x)=A+\ln x$.

Another way to think about the condition is in terms of coefficients of absolute risk aversion and absolute prudence, taking $g()$ to be analogous to the utility function. Than the sufficient condition is that coefficient of absolute prudence is larger than twice the coefficient of absolute risk aversion. Similar conditions arise in other matching settings, such as Newman (2007) and Theile and Wambach (1999).

We can also prove a partial converse, relating concave $r(x)$ to negative matching.

Claim 3 Suppose that $-\frac{g(x) g^{\prime \prime}(x)}{\left(g^{\prime}(x)\right)^{2}}$ is (weakly) decreasing and for the positively matched team the optimal effort level is interior. Then the conditions of Claim 1 hold, and thus it is optimal to sort the teams negatively.

Proof. For a positively matched team, the FOC from (2) is

$$
g^{\prime}(x)\left(1-\frac{2 \theta}{g^{\prime}(x)}\right)+g(x) \cdot \frac{2 \theta g^{\prime \prime}(x)}{\left(g^{\prime}(x)\right)^{2}}=0 .
$$

It can be simplified to

$$
\frac{g^{\prime}(x)}{\theta}=2\left(1-\frac{g(x) g^{\prime \prime}(x)}{\left(g^{\prime}(x)\right)^{2}}\right)
$$


or

$$
\frac{1}{w_{\theta}}=2\left(1-\frac{g\left(x_{\theta}\right) g^{\prime \prime}\left(x_{\theta}\right)}{\left(g^{\prime}\left(x_{\theta}\right)\right)^{2}}\right)
$$

If $-\frac{g(x) g^{\prime \prime}(x)}{\left(g^{\prime}(x)\right)^{2}}$ is decreasing, then the right hand side is decreasing in $x$; therefore Then if, for instance, higher $\theta$ leads to higher $x_{\theta}$ (making the RHS smaller), it must be the case that higher $\theta$ makes $w_{\theta}$ larger. But then higher inputs are associated with higher bonuses, and negative matching is optimal, a contradiction to the asserted positive matching.

In this case, we need that $r(x)$ is sufficiently concave in order to get negative matching. However, note that this does not mean that $g(x)$ must be terribly unusual; for instance, $g(x)=x^{\alpha}$ meets the requirement for all $\alpha$ strictly between zero and one. In the utility function language, the coefficient of absolute prudence, plus the ratio of $g^{\prime}(x)$ to $g(x)$, must be smaller than twice the coefficient of absolute risk aversion.

Intuitively, the convexity/concavity of the reward function function is important for matching predictions because it determines whether the differences in compensations are mostly driven by the differences in marginal costs or by the differences in effort levels. As we emphasized earlier, the relationship between the agent's cost of effort and his compensation is generally ambiguous: an agent with the lower cost would be asked to exert more effort, but is compensated less per unit of effort. The question, then, is how much greater a level of effort should be chosen for the low cost agent, and how much more cost that higher effort will necessitate. When the required reward is a very convex function of effort, it is prohibitively expensive to make effort substantially higher for the low cost agent, and therefore the main difference between the agents is not effort levels, but the required cost to provide a given effort level. As a result, the higher input agent (the low cost type) is paid less in the event of a success, and positive matching is optimal. By contrast, when the reward required to implement a given level of effort is a concave function, the low cost agent can be asked to give a great deal higher effort, overcoming the lower cost and implying that the low cost agent gets a greater reward in the event of a success. The first set of results implies that this case is exactly the one where negative matching is optimal.

It is also instructive to point out that Claims 2 and 3 establish sufficient conditions for super- and sub- modularity of the planner's profit function (under 
the assumption that solution is interior). This means that the mechanism described in our model illustrates how complementarities or anticomplementaries in the payoff function can endogenously arise due to the presence of moral hazard friction.

\section{Complete Characterization: Examples}

\subsection{Exponential Case}

To understand how non-interior cases impact the matching structure, we focus on the special case of $g(x)=\left(1-e^{-x}\right) / 2$. We know from the prior section that any interior case will always have the feature that matching is positive. We then

only have to consider the outcome for this interior, positive matched case, as compared with the possible corner solutions for both matching structures.

For the interior case, the planner's surplus from a team of type $\theta$ is found by solving

$$
\max _{x} 2 g(x)\left(1-2 \theta / g^{\prime}(x)\right)
$$

The first order condition is

$$
e^{-x}=4 \theta e^{x}
$$

which implies $e^{-x}=2 \sqrt{\theta}$ and the maximized payoff is

$$
1-4 \sqrt{\theta}+4 \theta
$$

It is also shown in the Appendix that, whenever positive matching structure is optimal, the second order condition for the positively matched teams is satisfied automatically.

\subsubsection{The effect of corners on matching predictions}

For comparison, consider a team with one team member giving zero effort. It is easy to show that the zero effort agent will always be the higher cost type, if the team is heterogenous. Therefore the planner must only choose an effort for the 
(weakly) lower type $\theta$, in order to solve

$$
\max _{x} g(x)\left(1-\theta / g^{\prime}(x)\right)
$$

In this case the first order condition is

$$
e^{-x}=2 \theta e^{x}
$$

so we have that $e^{-x}=\sqrt{2 \theta}$ for the agent that works $x>0$, and a maximized payoff of

$$
1 / 2-\sqrt{2 \theta}+\theta
$$

For a team with two like types, then, the decision to choose $x=0$ for one of them occurs at $\theta^{*}=\frac{3}{2}-\sqrt{2}$; for small $\theta$, both work, but for high $\theta$ only one works.

We know from the previous section that for this form of $g(x)$ negative matching may occur only if the interior solution is chosen. In the corner solution case the payoff is as computed in (6). It is immediate that if $\theta^{*}<l<h$, so that any team would have one worker providing zero effort, it is optimal that the planner chooses negative matching, so that a low quality type provides effort in both teams.

If, by contrast, $l<h<\theta^{*}$, positive matching has both with both agents on both teams working an interior amount. The alternative is negative matching with only the $l$ types working. The payoff in the positive case is

$$
1-4 \sqrt{l}+4 l+1-4 \sqrt{h}+4 h
$$

whereas the negative case has payoff

$$
2(1 / 2-\sqrt{2 l}+l)
$$

It is easily verified that the positive matching payoff is larger for all $l$ and $h$ below $\theta^{*}$.

The final case is the one where $l<\theta^{*}<h$. In this case, negative matching remains with the same payoff of

$$
2(1 / 2-\sqrt{2 l}+l)
$$


but positive matching gives a payoff of

$$
1-4 \sqrt{l}+4 l+1 / 2-\sqrt{2 h}+h
$$

since the team of two $h$ types has only one providing positive $x$. For fixed $l$, as $h$ rises, the payoff to the positive matching case falls until the team is no longer productive at all, and the payoff to the negative matching outcome remains constant. For large $h$ the negative case always dominates, and for $h$ near $\theta^{*}$ the positive case dominates. Therefore, for every $l$, there is a cutoff $h^{*}(l)$ such that negative matching is chose in $h$ is greater than $h^{*}(l)$. It is easy to verify that $h^{*}(l)$ is decreasing in $l$, with $h^{*}\left(\theta^{*}\right)=\theta^{*}$.

Intuitively, for fixed $l$, negative matching is associated with a sufficiently high cost for type- $h$ workers. When both types are sufficiently good, all agents give effort and the form of $g$ guarantees interior solutions. However, as the higher cost agent gets worse, the payoff from that team declines. Eventually the team of $h$ type moves to a corner solution where only one agent provides effort. Eventually the payoff from that team doesn't even justify its existence, and the principal does better by switching to positive matching.

Corner solutions here need not be interpreted as agents not working. It is simply that those agents don't contribute unobserved effort and receive bonus payments. Agents with $x=0$ could still be providing observable effort essential to the operation of the technology, and be compensated in accordance with their cost of effort. Here all those efforts and payments are fixed at zero, but none of the results require that those payments do not exist.

\subsubsection{Properties of positive versus negative matching}

If the firm owner were to put together mixed teams, the optimal efforts would solve

$$
\max _{x_{l}, x_{h}}\left(g\left(x_{l}\right)+g\left(x_{h}\right)\right)\left(1-l / g^{\prime}\left(x_{l}\right)-h / g^{\prime}\left(x_{h}\right)\right),
$$

which implies that

$$
e^{-x_{l}}=2 \sqrt{l} \text { and } \quad e^{-x_{h}}=2 \sqrt{h}
$$

Notice that one special feature of the exponential function is that workers' optimal effort levels (as well as rewards) do not depend on the matching structure. As 
a result, if the firm owner allowed the workers to organize in teams themselves, they would also end up forming positive matches (unless they can commit making side payments). The reason is that everyone, including low cost workers, would prefer to be teamed with another low cost worker because this would maximize the frequency of successful outcomes (and, hence, expected rewards).

If, on the other hand, the workers could commit to making side payments, they would prefer to form mixed teams in order to maximize the total rewards paid by the planner. In order to resolve the conflict between the workers' and his own matching preferences, the planner could transfer some utility to the agents and induce positive matching. However, recall that in this example efforts do not depend on the matching structure, hence the total surplus (measured as $\left.2 g\left(x_{l}\right)+2 g\left(x_{h}\right)-2 l x_{l}-2 h x_{h}\right)$ would also remain constant across different matching structures. Therefore, if we allowed agents to transfer utility to each other at no cost, the model with the exponential $g(x)$ would generate no matching predictions. In contrast, in the next suction we study a different example, in which the matching structure that maximizes the planner's profit also maximizes the total surplus.

\subsection{Example 2: Power function case}

Suppose that $g(x)=x^{\alpha}$, with $\alpha \in(0,1 / 2)$ and feasible effort $x \in[0,1 / 4] .{ }^{4}$ Then $-\frac{g(x) g^{\prime \prime}(x)}{\left(g^{\prime}(x)\right)^{2}}=\alpha(1-\alpha)$, which, by Claim 3, implies that the optimal matching structure is negative. From the first order conditions, the optimal effort levels satisfy

$$
\begin{aligned}
& \alpha x_{l}^{\alpha-1}=\frac{\theta_{l}}{\alpha}\left[1+\left(\frac{\theta_{l}}{\theta_{h}}\right)^{\frac{\alpha}{1-2 \alpha}}\right] \\
& \alpha x_{h}^{\alpha-1}=\alpha x_{l}^{\alpha-1}\left(\frac{\theta_{l}}{\theta_{h}}\right)^{\frac{\alpha-1}{1-2 \alpha}}=\frac{\theta_{h}}{\alpha}\left[1+\left(\frac{\theta_{h}}{\theta_{l}}\right)^{\frac{\alpha}{1-2 \alpha}}\right]
\end{aligned}
$$

In the Appendix it is verified that the solution to (7) also satisfies the second order condition as long as $\alpha<1 / 2$.

Notice also that if the workers are matched positively, the agents with marginal

\footnotetext{
${ }^{4}$ The restriction $x \leq 1 / 4$ is imposed to guarantee that $g\left(x_{l}\right)+g\left(x_{h}\right) \leq 1 / 2$.
} 
$\operatorname{cost} \theta$ would be asked to put effort $x$ which satisfies

$$
\alpha x^{\alpha-1}=2 \theta / \alpha .
$$

In this case the second order would obviously be satisfied.

\subsubsection{Some properties of the optimal contract (interior solution)}

(a) High-cost agent receives lower utility than the low-cost agent, independently of the matching structure

(i) Suppose that the workers are matched negatively. Then the difference between the worker's utilities is

$$
u_{l}-u_{h}=\left(g\left(x_{l}\right)+g\left(x_{h}\right)\right)\left(\frac{\theta_{l}}{g^{\prime}\left(x_{l}\right)}-\frac{\theta_{h}}{g^{\prime}\left(x_{h}\right)}\right)-\left(\theta_{l} x_{l}-\theta_{h} x_{h}\right) .
$$

Note that in general case we cannot say anything about the sign of the right hand side expression in (9). However, when $g(x)=x^{\alpha}$, it simplifies to

$$
u_{l}-u_{h}=\theta_{1} x_{1} \frac{1-\alpha}{\alpha}\left[1-\left(\frac{\theta_{l}}{\theta_{h}}\right)^{\frac{2 \alpha}{1-2 \alpha}}\right]
$$

which is positive if $\theta_{l}<\theta_{h}$ and $0<\alpha<1 / 2$.

(ii) When the workers are matched positively, the utility of a worker of type $\theta$ is given by

$$
u=2 g(x) \frac{\theta}{g^{\prime}(x)}-\theta x,
$$

which for the power function $g(x)=x^{\alpha}$ simplifies to

$$
u=2 x^{\alpha} \frac{\theta}{\alpha x^{\alpha-1}}-\theta x=2 \theta x \frac{1-\alpha}{\alpha}=2 \theta^{-\frac{\alpha}{1-\alpha}}\left(\frac{\alpha^{2}}{2}\right)^{\frac{1}{1-\alpha}} \frac{1-\alpha}{\alpha},
$$

and is decreasing in $\theta$.

(b) The utility of an agent declines as the quality of his partner improves. 
In a mixed match, the utility of the agent of type $\theta_{h}$ is given by

$$
u_{h}=\left(g\left(x_{h}\right)+g\left(x_{l}\right)\right) \frac{\theta_{h}}{g^{\prime}\left(x_{h}\right)}-\theta_{h} x_{h} .
$$

Improvements in the quality of the partner affect the agent's surplus in two ways. On one hand, when the effort cost of the partner declines, he will be asked to work more, which would increase the frequency of accidental payments. On the other hand, the firm owner would want to increase the partner's contribution to the total team output, and therefore would reduce the effort assignment of the agent whose cost did not adjust, thus lowering his compensation. In general, it is not clear which of the two effects will dominate. However, a clear prediction can be made for the power function $g(x)$.

When $g(x)=x^{\alpha}, u_{h}$ can be expressed as

$$
u_{h}=\left(x_{h}^{\alpha}+x_{l}^{\alpha}\right) \frac{\theta_{h}}{\alpha x_{h}^{\alpha-1}}-\theta_{h} x_{h}=\frac{\theta_{h}}{\alpha}\left[1-\alpha+\left(\frac{x_{l}}{x_{h}}\right)^{\alpha}\right] .
$$

Using (7), we can express it as

$$
u_{h}=\alpha^{1-\alpha}\left(\frac{\alpha}{\theta_{h}}\right)^{\frac{\alpha}{1-\alpha}} \frac{1-\alpha+\left(\frac{\theta_{h}}{\theta_{l}}\right)^{\frac{\alpha}{1-2 \alpha}}}{\left(1+\left(\frac{\theta_{h}}{\theta_{l}}\right)^{\frac{\alpha}{1-2 \alpha}}\right)^{\frac{1}{1-\alpha}}}
$$

which is decreasing in $\left(\frac{\theta_{h}}{\theta_{l}}\right)^{\frac{\alpha}{1-2 \alpha}}$ and, for $\alpha \in(0,1 / 2)$, increasing in $\theta_{l}$.

Corollary 4 The firm owner and the low-cost worker prefer negative matching structure, but the high-cost worker prefers positive matching structure.

This observation raises a question of whether negative team sorting could be sustained if teams were formed by the workers, not by the firm owner. To answer this question, one would have to compare the total surplus in different matching structures. We were not able to implement such comparison analytically, but our numerical results suggest that the total surplus is higher when the teams are matched negatively (at least this is true for 

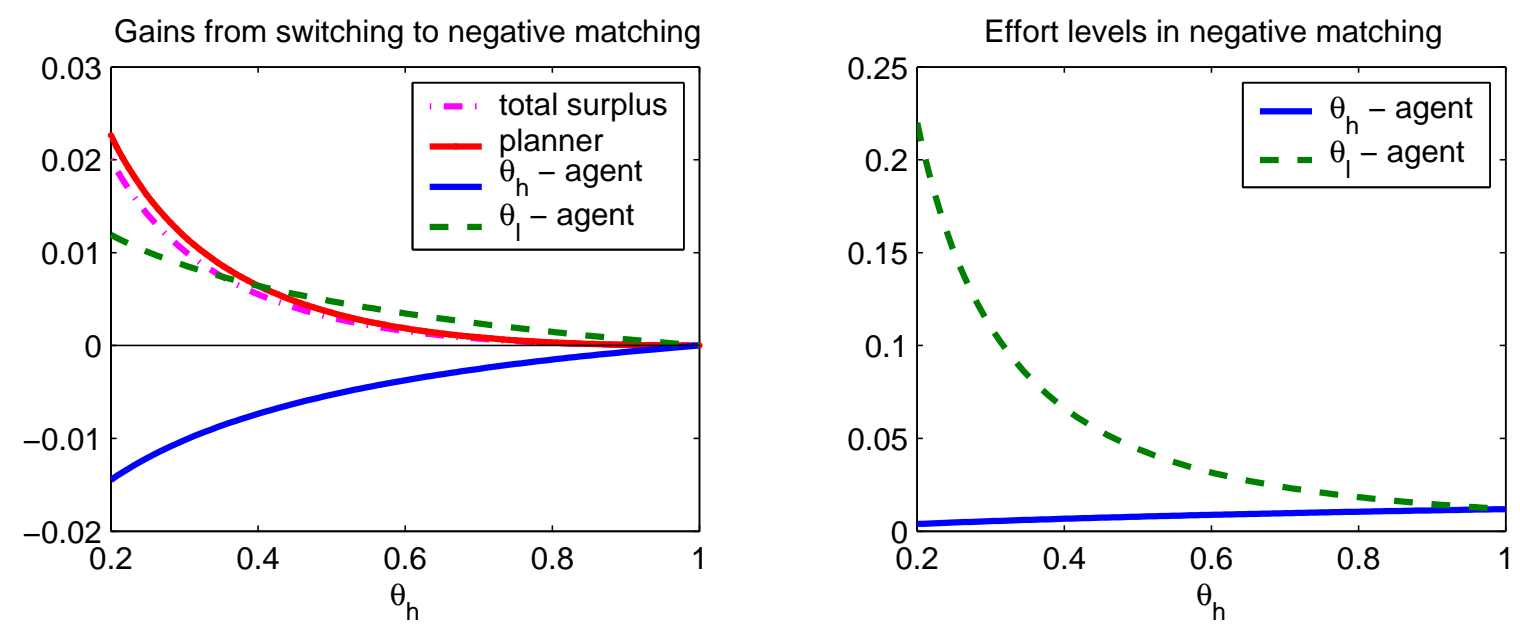

Figure 3: Properties of the optimal contract for $g(x)=x^{\alpha}$, where $\alpha=0.3, \theta_{h}=1$ and $\theta_{l} \in(0.2,1)$.

any parameter values that we tried). For instance, the left panel on Figure 5.2.1 compares the utilities, the planner's profit and the total surplus under positive and negative matching for different values of $\theta_{l} \in\left(0, \theta_{h}\right)$.

\section{$6 \quad$ Extensions}

\subsection{Larger teams}

Although we focused on teams of two, many of our results can easily be extended to larger teams. For instance, the results on interior solutions extend directly the case of teams of greater than two. In other words, when solutions are interior, the matching structure can be described by the shape of the $g(x)$ function using exactly the conditions described in that section.

For that case, then, team size can only impact the matching structure through the impact it has on choosing corners. As teams grow, there will be a tendency to move toward corner solutions, since there will be greater accidental payment for any level of bonus promised to the agent, coming from the agents relatively small role in the larger and larger team. As a result, we may see switches from positive to negative matching as in the exponential case above, where the switch is driven by a switch from interior solutions to corners as team size rises. 


\subsection{Relaxing Limited Liability}

Our results were all generated in the context of a model where moral hazard frictions arise due to limited liability. Since agents are risk neutral, limited liability is essential to this friction. It is, however, instructive to point out that our results are not driven by the restriction of non-negativity of rewards. Instead, we could require that $w_{i} \geq \underline{w}_{i}$, where $\underline{w}_{i}$ s are negative (and potentially different across different types), and our result would still apply as long as this constraint is binding for all the workers in all the matching structures, i.e. if $\underline{w}_{i} \mathrm{~s}$ is not too small. When $\underline{w}$ becomes sufficiently low, the workers' individual rationality constraints would become binding in all the matches for all the types, effort allocation would coincide with the first best, and matching would become irrelevant. The cases with intermediate values of $\underline{w_{i}} s$, for which individual rationality constraint binds occasionally (for some types in some matches) are hard to characterize in general. Our preliminary analysis suggests that our main results are preserved for some of those cases, but it seems impossible to make analytical prediction in all of them.

Perhaps, a better way to understand whether limited liability is the major driving force of our results would be to consider a model, in which the workers are risk averse and the compensation provided by the planner is the only source of the workers' consumption. Under this modification, the decision problem of the firm owner becomes

$$
\begin{aligned}
\max _{x_{l}, x_{h}, r_{l}, r_{h}} & {\left[g\left(x_{i}\right)+g\left(x_{j}\right)\right]\left(1-r_{l}^{1}-r_{h}^{1}\right)-\left[1-\left(g\left(x_{i}\right)+g\left(x_{j}\right)\right)\right]\left(r_{l}^{0}+r_{j}^{0}\right) } \\
\text { s.t. } & x_{i}=\arg \max _{x}\left\{\left[g\left(x_{i}\right)+g\left(x_{j}\right)\right] u\left(r_{i}^{1}\right)+\left[1-g\left(x_{i}\right)-g\left(x_{j}\right)\right] u\left(r_{i}^{0}\right)-\theta_{i} x_{i}\right\}, \quad i=l, h \\
& {\left[g\left(x_{i}\right)+g\left(x_{j}\right)\right] u\left(r_{i}^{1}\right)+\left[1-g\left(x_{i}\right)-g\left(x_{j}\right)\right] u\left(r_{i}^{0}\right)-\theta_{i} x_{i}=\underline{u}, \quad i=l, h . }
\end{aligned}
$$

As in our benchmark model, the combination of rewards and efforts that are incentive compatible for one matching structure would also be incentive compatible for another matching structure. Thus the planner might have incentives to rearrange the teams in order to minimize the 'accidental' bonuses, which are in the heart of our previous analysis. ${ }^{5}$ However, when teams are rearranged, the

\footnotetext{
${ }^{5}$ Notice that in this modification the variable in interest is the difference $r_{i}^{1}-r_{i}^{0}$ between the payments in the event and success and failure.
} 


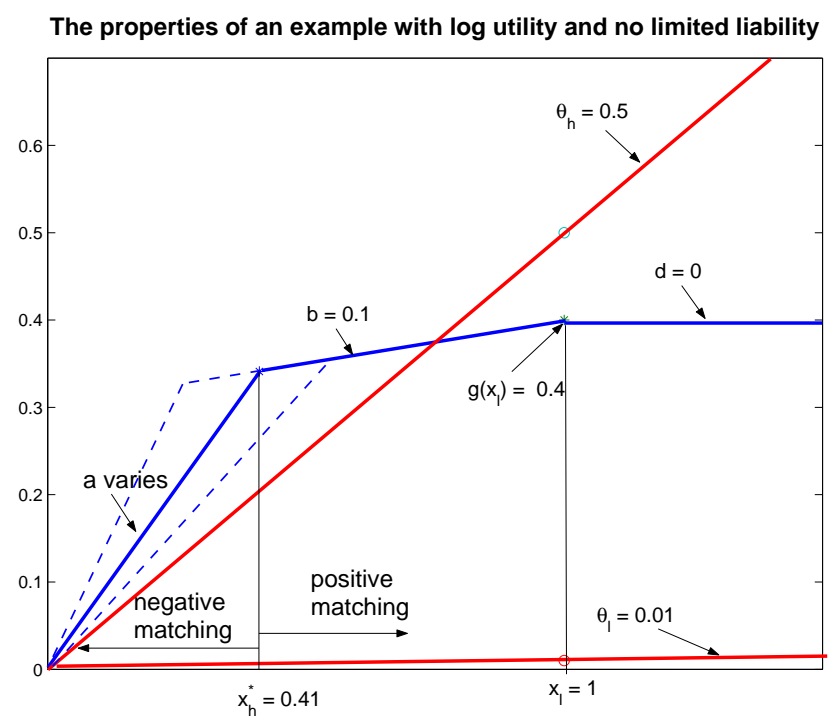

Figure 4: Matching predictions in the model with piecewise-linear $g(x)$ and logarithmic utility, $\underline{u}=\ln (0.05)$.

rewards would have to be adjusted to satisfy individual rationality constraints. For instance, while switching from the negative to the positive matching, the payments to the high-cost type would have to increase, but the payments to the low-cost type would fall (provided that the planner does not adjust the effort levels and therefore, keeps $u\left(r_{i}^{1}\right)-u\left(r_{i}^{0}\right)$ constant). We attempted to derive a set of conditions, under which the former change in payments is smaller in magnitude than the latter (thereby implying that switching to the positive matching would be beneficial), but the concavity of $u(c)$ substantially complicates the analysis, and we were not able to obtain any analytical predictions.

Thus, as a first step, we numerically solved an example with piecewise-linear $g(x)$ (introduced in section 4.2) and logarithmic utility. We found that the the model with risk aversion and binding individual rationality constraints generates predictions that are qualitatively similar to the ones obtained in our benchmark model: negative matching is optimal when $x_{h}$ is low, and positive matching is optimal when $x_{h}$ is high. Figure 4 illustrates an example for a particular set of parameters and reports the cutoff level $x_{h}^{*}$ at which the planner is indifferent between the two matching structures. It also turns out that this cutoff level is greater than the one that would be obtained in the model with risk neutrality and 
binding limited liability (where $\left.x_{h}^{*}=0.06\right)^{6}$, suggesting that, at least for some specifications, relaxing limited liability and introducing risk aversion may favor negative matching.

\subsection{Matching in partnerships}

In our model the principal's ex post profit is always positive, which suggests that the workers could achieve more efficient effort allocation if they formed partnerships and split the total output in between themselves. ${ }^{7}$ In this section we show numerically that both negative and positive matching may arise as the equilibrium outcome if such partnerships are formed.

Suppose that a partnership of two agents agrees that the first agent receives share $s_{1}$ of total output, and the second agent gets the rest. Given this agreement, the incentive compatible efforts are

$$
\begin{aligned}
& g^{\prime}\left(x_{1}\right) s_{1}=\theta_{1} \\
& g^{\prime}\left(x_{2}\right)\left(1-s_{1}\right)=\theta_{2} .
\end{aligned}
$$

Correspondingly, we can determine the pair of utilities derived by the agents for a given sharing rule:

$$
\begin{aligned}
& W\left(s_{1}\right)=\left(g\left(x_{1}\right)+g\left(x_{2}\right)\right)\left(1-\frac{\theta_{1}}{g^{\prime}\left(x_{1}\right)}\right)-\theta_{2} x_{2} \\
& v\left(s_{1}\right)=\left(g\left(x_{1}\right)+g\left(x_{2}\right)\right) \frac{\theta_{1}}{g^{\prime}\left(x_{1}\right)}-\theta_{1} x_{1} .
\end{aligned}
$$

By varying the sharing rule $s_{1}$ we obtain the frontier $W(v)$ of values delivered by the contract. This frontier is not linear ${ }^{8}$, implying that this model can be classified as the model with non-transferable utility studied in the matching literature. Legros and Newman (2007) provide sufficient conditions for positive (negative) equilibrium matching in this environment.

\footnotetext{
${ }^{6}$ Such $x_{h}^{*}$ is computed to equalize the rewards of the two types, $\theta_{h} / a=\theta_{l} / b$.

${ }^{7}$ The first best efforts satisfy $g^{\prime}\left(x_{i}\right)=\theta_{i}$, while in our model $g^{\prime}\left(x_{i}\right) w_{i}=\theta_{i}$, where $w_{l}+w_{h}<1$. Hence the total surplus might increase if the agents bypass the firm owner and obtain $w_{l}+w_{h}=1$ by forming a partnership.

${ }^{8}$ Not that $W(v)$ is not monotone in $s_{1}$, so we would focus on the intermediate values of $s_{1}$, for which $W(v)$ is decreasing.
} 

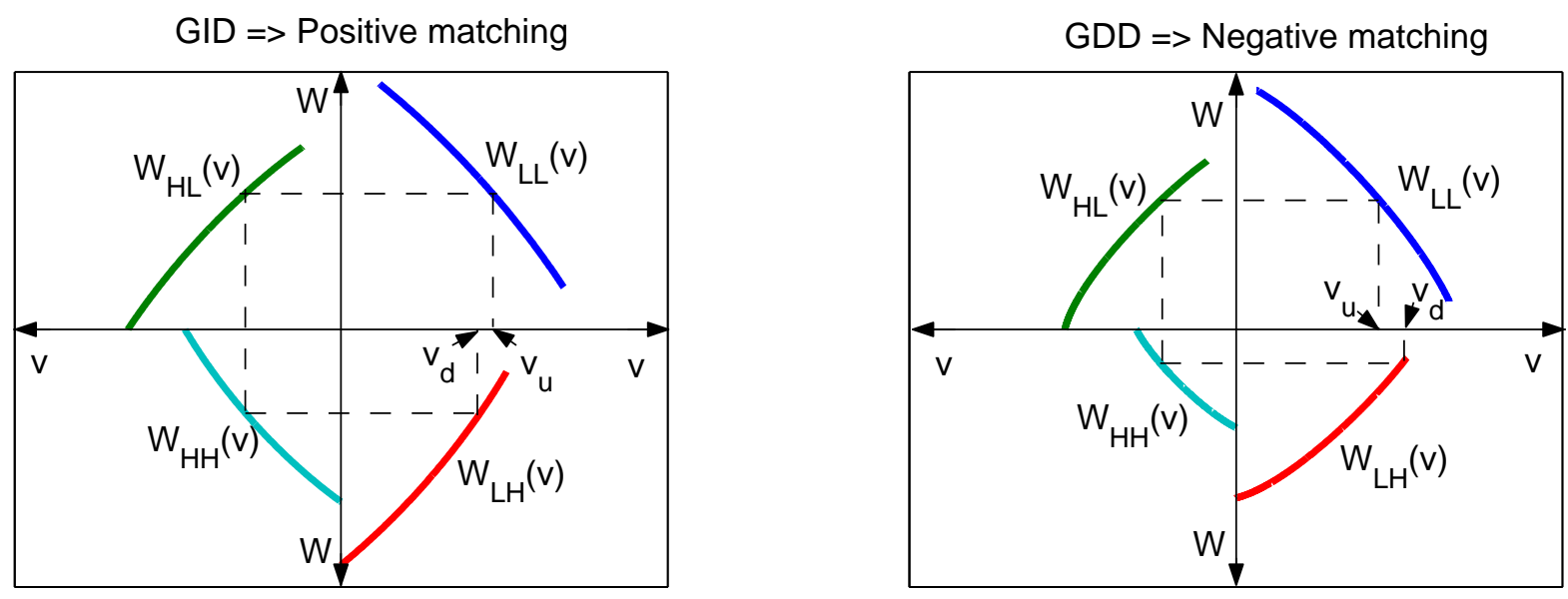

Figure 5: Properties of the optimal contract for $g(x)=x^{\alpha}$, where $\alpha=0.3, \theta_{h}=1$ and $\theta_{l} \in(0.2,1)$.

Denote by $W_{i j}(v)$ the frontier for the pair of agents with marginal costs $\theta_{i}$ and $\theta_{j}$ respectively, and by $v_{i j}^{-1}(W)$ the inverse of this frontier. Suppose that $\theta_{H}>\theta_{L}$. Legros and Newman (2007) show that

- if generalized increasing differences condition (GID) holds, i.e. for any $v$ at which $W_{L L}(v)$ is defined and decreasing the following condition holds:

$$
v_{u}(v)=v_{L L}^{-1}\left(W_{H L}(v)\right) \geq v_{L H}^{-1}\left(W_{H H}(v)\right)=v_{d}(v),
$$

then the equilibrium allocation is characterized by positive assortative matching;

- if generalized decreasing differences condition (GDD) holds, for any $v$ at which $W_{L L}(v)$ is defined and decreasing the following condition holds:

$$
v_{u}(v)=v_{L L}^{-1}\left(W_{H L}(v)\right) \leq v_{L H}^{-1}\left(W_{H H}(v)\right)=v_{d}(v)
$$

then the equilibrium allocation exhibits negative assortative matching.

The examples of frontiers satisfying GID and GDD conditions are illustrated on the two plots of Figure 5. Intuitively, suppose that a high-cost and a low-cost agents compete for being paired with the low-cost partner, conditional that the other high-cost agent is promised utility $v$. Then GID condition guarantees the 

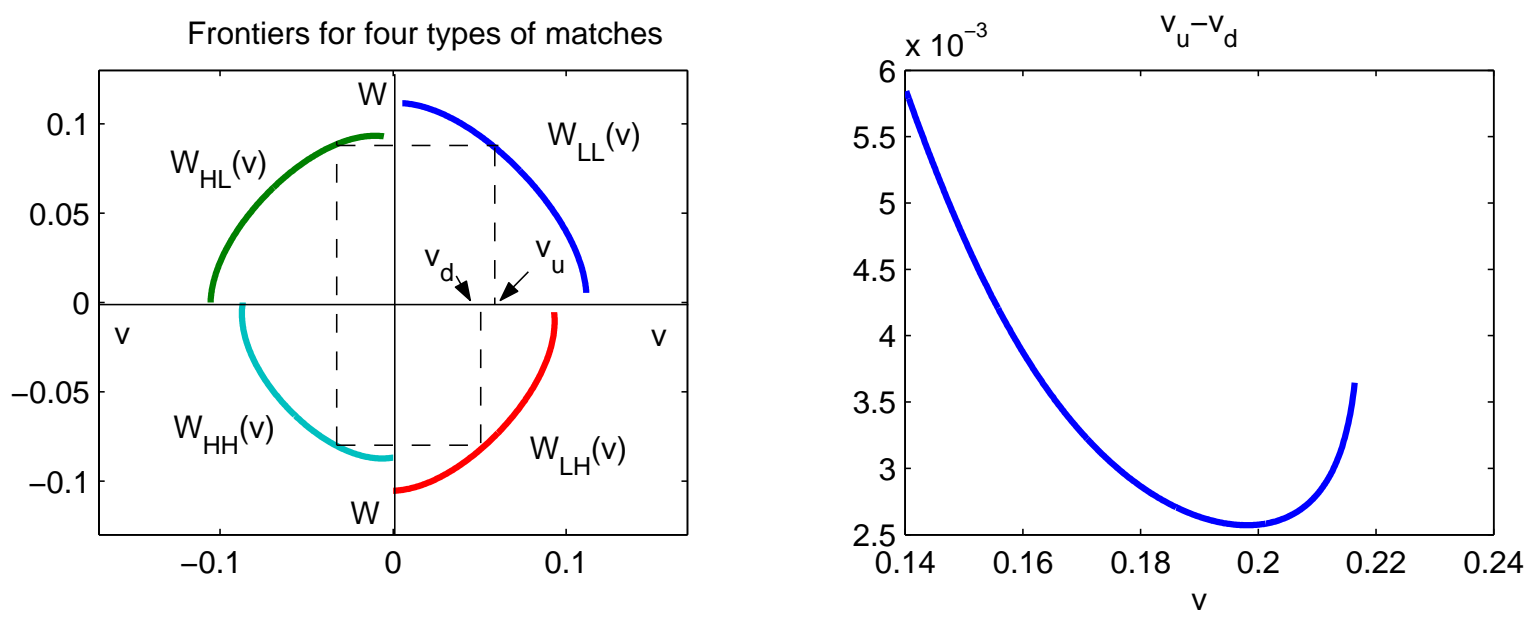

Figure 6: Frontiers and $v_{u}(v)-v_{d}(v)$ for $g(x)=\left(1-e^{-x}\right) / 2, \theta_{l}=0.1, \theta_{h}=0.11$. Since $v_{u}(v)-v_{d}(v)>0$ for all $v$, GID condition holds and positive matching obtains in equilibrium.

low-cost agent will be able to outbid the high-cost agent - because the former would be willing to agree on any sharing rule which gives the low-cost agent up to $v_{u}$, while the latter can promise to the low-cost agent only up to $v_{d}<v_{u}$. The argument for why GDD condition leads to negative matching is just the opposite.

Unfortunately, we were not able to analytically characterize the frontiers defined by (13) and (14), even for particular functional forms of $g(x)$. Therefore, we ran a large number of numerical exercises for $g(x)=\left(1-e^{-x}\right) / 2$ and for $g(x)=x^{\alpha}, \alpha \in(0,1)$. Figures 6 and 7 illustrate two examples of the solutions for these functional forms. Remarkably, for all the parameter values that we tried, we found GID condition is satisfied when $g(x)$ is exponential and GDD condition holds if $g(x)$ is a power function, implying positive matching in the former case and negative in the latter. Recall that in our benchmark model exactly the same matching predictions are obtained for these functional forms, suggesting that our intuition could perhaps be applicable to a broader class of models.

\section{Conclusion}

We develop a model of team production where, in the absence of information frictions, there is no reason for any matching structure to prevail. Once we add a 

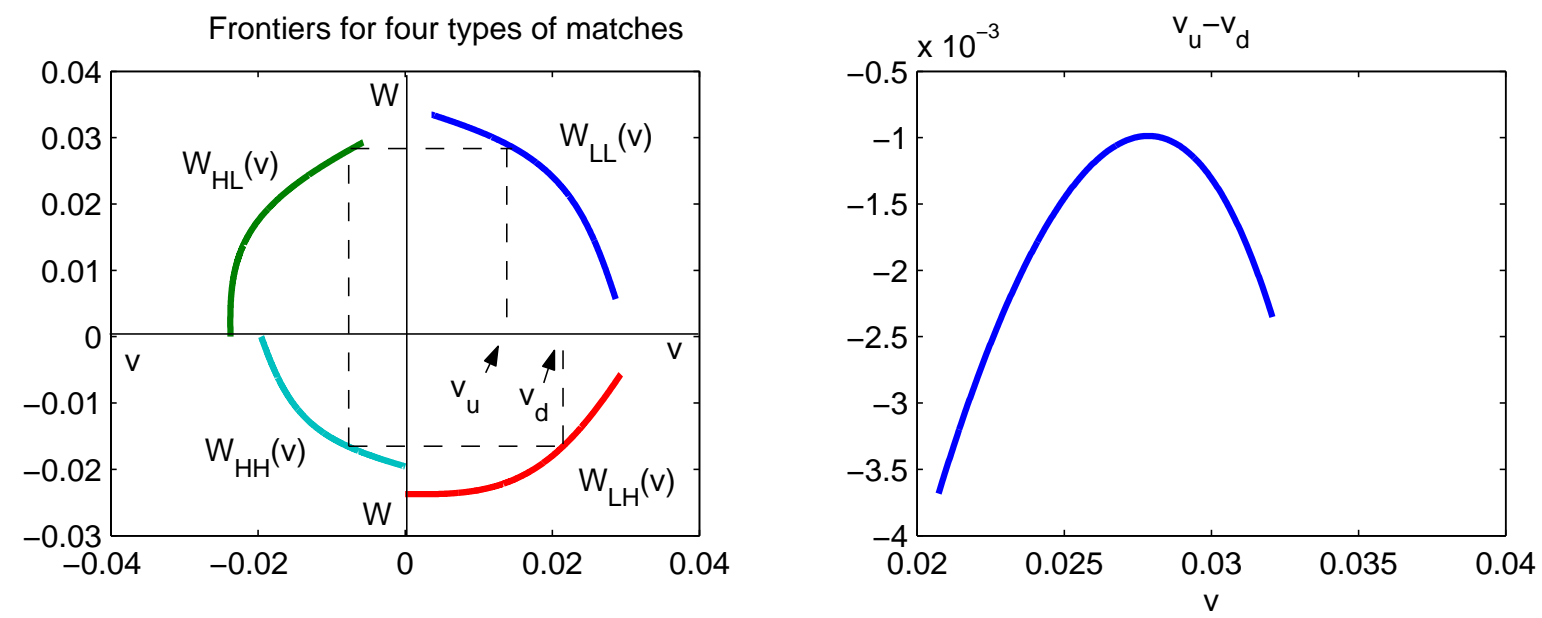

Figure 7: Frontiers and $v_{u}(v)-v_{d}(v)$ for $g(x)=x^{0.55}, \theta_{l}=3, \theta_{h}=4$. Since $v_{u}(v)-v_{d}(v)<0$ for all $v$, GDD condition holds and negative matching obtains in equilibrium.

team moral hazard problem, there is typically a non-trivial matching decision for the principal. We formulate the solution to this decision in three ways. First we show that the decision is directly linked to the relationship between inputs across agents of different types, and the bonus they received. Second, we show that the structure is related to the shape of the relationship between an individual agents input and the team probability of success, in a way that is familiar from other matching structures that do not include a team component. Finally, for a special case, we show that the matching structure can depend on the way in which corner solutions arise. Corner solutions can switch the matching structure from positive to negative matching.

\section{References}

[1] Becker, Gary, A Theory of Marriage: Part I, Journal of Political Economy, Vol 81, no. 4, 1973, pp.813-846

[2] Hamilton, Barton H. , Jack A. Nickerson, and Hideo Owan, Team Incentives and Worker Heterogeneity: An Empirical Analysis of the Impact of Teams on Productivity and Participation Journal of Political Economy 2003 111:3, 465-497 
[3] Holmstrom, Bengt, Moral Hazard in Teams, The Bell Journal of Economics, Vol. 13, No. 2. (Autumn, 1982), pp. 324-340.

[4] Kaya, Ayca, Two-sided Matching with Private Information, Mimeo.

[5] Kremer, Michael. The O-Ring Theory of Economic Development, The Quarterly Journal of Economics, Volume 108, No. 3, 1993, pp.551-575.

[6] Legros, Patrick and Andrew F. Newman, Monotone Matching in Perfect and Imperfect Worlds, Review of Economic Studies Vol 69 NO: 4, 2002, PG: 925942.

[7] Legros, Patrick and Andrew F. Newman, Beauty Is a Beast, Frog Is a Prince: Assortative Matching with Nontransferabilities, Econometrica, Volume 75 (4), 2007, pp. 1073 - 1102.

[8] Andrew F. Newman, Risk-bearing and entrepreneurship, Journal of Economic TheoryVolume 137, Issue 1, November 2007, Pages 11-26.

[9] Andrea Prat, Should a team be homogeneous?, European Economic ReviewVolume 46, Issue 7, July 2002, Pages 1187-1207.

[10] Shimer, Robert and Lones Smith, Assortative Matching and Search, Econometrica, Volume 68, No. 2, 2003, pp. 343-369.

[11] Henrik Thiele, Achim Wambach, Wealth Effects in the Principal Agent Model, Journal of Economic TheoryVolume 89, Issue 2, , December 1999, Pages 247-260.

\section{Appendix}

\subsection{Second order conditions for positively matched teams}

If both workers in the team have marginal cost $\theta$, then the planner's profit is given by

$$
\Pi(x)=2 g(x)\left(1-\frac{2 \theta}{g^{\prime}(x)}\right) .
$$


Correspondingly,

$$
\Pi^{\prime}(x)=2 g^{\prime}(x)\left(1-\frac{2 \theta}{g^{\prime}(x)}\right)+4 \theta g(x) \frac{g^{\prime \prime}(x)}{\left(g^{\prime}(x)\right)^{2}},
$$

and

$$
\Pi^{\prime \prime}(x)=2 g^{\prime \prime}(x)-2 \theta\left(g(x) \frac{-g^{\prime \prime}(x)}{\left(g^{\prime}(x)\right)^{2}}\right)_{x}^{\prime} .
$$

Therefore, the second order condition is satisfied if $g(x) \frac{-g^{\prime \prime}(x)}{\left(g^{\prime}(x)\right)^{2}}$ is increasing (whenever the optimal matching structure is positive), but may be violated otherwise.

\subsection{Second order conditions for negatively matched teams}

The planner's profit in the team consisting of two different workers, with marginal $\operatorname{costs} \theta_{l}$ and $\theta_{h}$, is given by

$$
\Pi\left(x_{l}, x_{h}\right)=\left(g\left(x_{l}\right)+g\left(x_{h}\right)\right)\left(1-\frac{\theta_{l}}{g^{\prime}\left(x_{l}\right)}-\frac{\theta_{h}}{g^{\prime}\left(x_{h}\right)}\right) .
$$

Thus

$$
\begin{aligned}
& \frac{\partial \Pi\left(x_{l}, x_{h}\right)}{\partial x_{l}}=g^{\prime}\left(x_{l}\right)\left(1-\frac{\theta_{l}}{g^{\prime}\left(x_{l}\right)}-\frac{\theta_{h}}{g^{\prime}\left(x_{h}\right)}\right)+\theta_{l} \frac{g^{\prime \prime}\left(x_{l}\right)}{g^{\prime}\left(x_{l}\right)^{2}}\left(g\left(x_{l}\right)+g\left(x_{h}\right)\right), \\
& \frac{\partial^{2} \Pi\left(x_{l}, x_{h}\right)}{\partial x_{l}^{2}}=g^{\prime \prime}\left(x_{l}\right)\left(1-\frac{\theta_{h}}{g^{\prime}\left(x_{h}\right)}\right)-\theta_{l}\left(\frac{-g^{\prime \prime}\left(x_{l}\right)}{g^{\prime}\left(x_{l}\right)^{2}}\left(g\left(x_{l}\right)+g\left(x_{h}\right)\right)\right)_{x_{l}}^{\prime}
\end{aligned}
$$

Note that, as for the positively matched teams, $\frac{\partial^{2} \Pi\left(x_{l}, x_{h}\right)}{\partial x_{l}^{2}}<0$ if positive matching is optimal but the second order condition may be violated if $g(x) \frac{-g^{\prime \prime}(x)}{\left(g^{\prime}(x)\right)^{2}}$ is decreasing (i.e., if the negative matching structure is optimal).

Since the problem is symmetric, the same is true for $\frac{\partial^{2} \Pi\left(x_{l}, x_{h}\right)}{\partial x_{h}^{2}}$.

The cross-derivative is

$$
\frac{\partial^{2} \Pi\left(x_{l}, x_{h}\right)}{\partial x_{l} \partial x_{h}}=\theta_{h} g^{\prime}\left(x_{l}\right) \frac{g^{\prime \prime}\left(x_{h}\right)}{\left(g^{\prime}\left(x_{h}\right)\right)^{2}}+\theta_{l} g^{\prime}\left(x_{h}\right) \frac{g^{\prime \prime}\left(x_{l}\right)}{\left(g^{\prime}\left(x_{l}\right)\right)^{2}},
$$


which, by the first order conditions, can be simplified to

$$
\frac{\partial^{2} \Pi\left(x_{l}, x_{h}\right)}{\partial x_{l} \partial x_{h}}=2 \theta_{h} g^{\prime}\left(x_{l}\right) \frac{g^{\prime \prime}\left(x_{h}\right)}{\left(g^{\prime}\left(x_{h}\right)\right)^{2}}=2 \theta_{l} g^{\prime}\left(x_{h}\right) \frac{g^{\prime \prime}\left(x_{l}\right)}{\left(g^{\prime}\left(x_{l}\right)\right)^{2}} .
$$

Unfortunately, we cannot say anything conclusive regarding the sign of

$$
\frac{\partial^{2} \Pi\left(x_{l}, x_{h}\right)}{\partial x_{l}^{2}} \frac{\partial^{2} \Pi\left(x_{l}, x_{h}\right)}{\partial x_{h}^{2}}-\left(\frac{\partial^{2} \Pi\left(x_{l}, x_{h}\right)}{\partial x_{l} \partial x_{h}}\right)^{2},
$$

even if the positive matching is optimal. However, we can verify that the second order conditions hold for the particular functional forms of $g(x)$ considered below.

\subsection{Second order conditions for the power function}

Plugging $g(x)=x^{\alpha}$ into (22) and (24), we obtain that

$$
\frac{\partial^{2} \Pi\left(x_{l}, x_{h}\right)}{\partial x_{l}^{2}}=\alpha(\alpha-1) x_{l}^{\alpha-2}<0,
$$

and

$$
\frac{\partial^{2} \Pi\left(x_{l}, x_{h}\right)}{\partial x_{l} \partial x_{h}}=2(\alpha-1) \theta_{l} x_{l}^{\alpha} x_{h}^{\alpha-1}=2(\alpha-1) \theta_{h} x_{h}^{\alpha} x_{l}^{\alpha-1} .
$$

Correspondingly,

$$
\begin{aligned}
\frac{\partial^{2} \Pi\left(x_{l}, x_{h}\right)}{\partial x_{l}^{2}} \frac{\partial^{2} \Pi\left(x_{l}, x_{h}\right)}{\partial x_{h}^{2}}-\left(\frac{\partial^{2} \Pi\left(x_{l}, x_{h}\right)}{\partial x_{l} \partial x_{h}}\right)^{2}= \\
\quad=\alpha^{2}(1-\alpha)^{2} \frac{x_{l}^{\alpha-1} x_{h}^{\alpha-1}}{x_{l} x_{h}}-4 \theta_{l} \theta_{h}(\alpha-1)^{2} \frac{1}{x_{l} x_{h}}= \\
\quad=\frac{(1-\alpha)^{2}}{x_{l} x_{h}} \theta_{l} \theta_{h}\left[\frac{1}{\alpha^{2}}\left(1+\left(\frac{\theta_{l}}{\theta_{h}}\right)^{\frac{\alpha}{1-2 \alpha}}\right) \cdot\left(1+\left(\frac{\theta_{h}}{\theta_{l}}\right)^{\frac{\alpha}{1-2 \alpha}}\right)-4\right],
\end{aligned}
$$

which is definitely positive if $\alpha \in(0,1 / 2]$. 